\title{
The link between structural connectivity and neurocognition illustrated by focal epilepsy
}

\author{
Elise Roger ${ }^{1}$, Laurent Petit ${ }^{2}$, Marcela Perrone-Bertolotti ${ }^{1}$, \\ Anne-Sophie Job $^{3}$, Lorella Minotti ${ }^{3}$, Philippe Kahane ${ }^{3}$, \\ Monica Baciu ${ }^{1}$ \\ ${ }^{1}$ LPNC UMR 5105, CNRS, Univ. Grenoble Alpes, Grenoble \\ 2 GIN-IMN, UMR 5293, CEA/CNRS, Université de Bordeaux, Bordeaux \\ ${ }^{3}$ GIN, U836 UGA/INSERM “Synchronisation et modulation des réseaux neuronaux \\ dans l'épilepsie", Grenoble University Hospital, Grenoble, France
}

Received October 26, 2017; Accepted December 21, 2017

\begin{abstract}
Increasing attention is being paid to the assessment of white matter properties and its structural connectivity, both in healthy subjects and patients with cerebral lesions. Within this framework, new neurocognitive models based on hodological properties have been developed under a connectomic perspective in order to explain substrates and cognitive mechanisms related to cerebral functions such as language and memory. With regards to focal and drug-resistant epilepsy conceived as a network disorder, new insights in terms of structural connectivity have led to significant advances in epilepsy research, concerning fundamental research (neurocognitive mechanisms of plasticity) and clinical application (optimization of decision making for curative surgery). We believe that such findings in the literature, focused on the role of white matter in cerebral functioning in relation to neurocognition, may be helpful for both researchers and clinicians working in the field of epilepsy.
\end{abstract}

Key words: connectivity, white matter, hodology, focal epilepsy, plasticity, cognition

Correspondence:

Monica Baciu

Laboratoire de psychologie

et neurocognition,

UMR CNRS 5105,

Université Grenoble Alpes,

BP 4738040 Grenoble,

Cedex 09 France

<monica.baciu@univ-grenoble-alpes.fr>

\section{Structural connectivity and neurocognition: general information}

Structural connectivity refers to the study of the anatomical features of brain networks in terms of subcortical white matter (WM) that underlies interconnections between different cortical regions. Within the current context of growing interest with a connectomic perspective, many MRI methods and parameters are now available to assess WM anatomical properties including architecture, spatial trajectory, density or integrity (Sporns, 2013). These structural properties, investigated in relation to cognitive functions and behavioral performance, allows the development of more 
complex neurocognitive models and to supplant the traditional localizationist view of cognitive functioning for a more realistic hodological view (hodology, from "hodos" meaning "pathway" in Greek) (Duffau, 2017). A distinction is usually made between the macroscale and meso-microscale based on specific WM features. The macroscopic level refers to the global morphology of bundles (e.g. length, trajectory, or spatial disposition), whereas the meso-microscopic level (millimetre scale) refers to WM bundle properties that are not directly visible to the naked eye (e.g. tissue composition and integrity). The analysis of the meso-microscopic level, in a non-invasive way, can be carried out using MRI by exploiting various parameters derived from diffusion image processing (e.g. a diffusion tensor model [DTI]). At a more macroscopic level, the fibres can be modelled by tractographic reconstruction (deterministic or probabilistic tractography) on the basis of diffusion data (see educational slides [additional supplementary material] for a more detailed description and illustration of these major methods and techniques derived from diffusion MRI).

\section{Levels of structural connectivity and plasticity potential}

\section{Macroscopic level}

Macroscopically, WM fibres organise into clusters that form extensive communication pathways, classified as: - projection fibres (e.g. corona radiata, internal capsule, cortico-spinal tracts) which connect the cerebral cortex to the basal ganglia, brainstem, and grey matter of the spinal cord;

- commissural fibres (corpus callosum, anterior and posterior commissures, hippocampal commissures) which enable connections between regions of the left and right hemispheres;

- and association fibres which facilitate intrahemispheric connections and interconnect the various cortical regions within a given hemisphere; short fibres (U-shaped) interconnect adjacent gyri, whereas long fibre bundles interconnect the various lobes within a given hemisphere (see figure 1 for an example of long association fascicles).

Disconnection surgery and callosotomy (neurosurgical disconnections on the corpus callosum in patients with epilepsy) are associated with significant cognitive deficits (Glickstein and Berlucchi, 2008), suggesting that the WM fibres are essential for cognition. Indeed, large WM lesions are accompanied by severe behavioral and cognitive deficits and less favourable prognosis (e.g. Kiran, 2012; Lunven et al., 2015). Hence, neurosurgeons now make every possible effort to preserve the connections of the main networks and resections are no longer limited to certain cortical areas traditionally considered as crucial (eloquent areas); instead, the impact on WM bundles is avoided. A number of data support the idea that the plastic potential of WM macrostructure is minimal, suggesting low inter-individual variability and weak reorganization capacity of the WM. For instance, WM bundles in adults are similar to those in young children, which even connect cortical regions that have yet to mature (Dubois et al., 2009). Furthermore, studies performed on congenitally deaf cats show that these cats have the same basis of structural connectivity as cats with normal hearing (Lomber et al., 2010). No large-scale reorganization occurs in terms of anatomical association with the pathology. Interestingly, it also emerged from this study that over-developed abilities for peripheral vision and movement detection (the visual dorsal pathway) in deaf cats might be attributable to cross-modal functional plasticity, suggesting that unused analogous structures (the auditory dorsal pathway) are colonised by visual functions. Taken as a whole, these findings support the concept that -despite some of the variability within similar structures- the WM wiring is closely comparable in all subjects and goes hand in hand with the preservation of functional specificities (intrinsic characteristics). This echoes the concept of the "minimal common brain", described by lus et al. (2011) as an invariable constituent of the central nervous system based on severe constraints imposed by the cerebral architecture itself. Indeed, the major fascicles are composed of convergent fibres that traverse narrow spaces (WM capsules and stems; Hau et al., 2016) delimited by anatomical boundaries (gyri, sulci, ventricles, and grey nuclei), thus precluding the occurrence of major morphological variations (Duffau, 2017).

\section{Meso-microscopic level}

Mesoscopically, though relatively well structured, the cerebral WM exhibits a complex microstructural anatomy composed of axonal fibres and glial cells (mainly oligodendrocytes, astrocytes and microglia). Advances in the field of neuro-histology have allowed us to study myelination at various stages and suggests that the microstructure of the WM is dynamic. Recent studies have shown that the number of myelinated axons within bundles varies and all axons are not myelinated in the same way (Walhovd et al., 2014). Histologically, the myelin sheath thickness is quantified by using the myelin g-ratio. For many years, myelin thickness was thought to be a simple linear phenomenon determined by axonal diameter (Tomassy et al., 2014), which meant that the larger the axon, the more myelinated it would be. However, this correlation appears to be not so obvious, and 


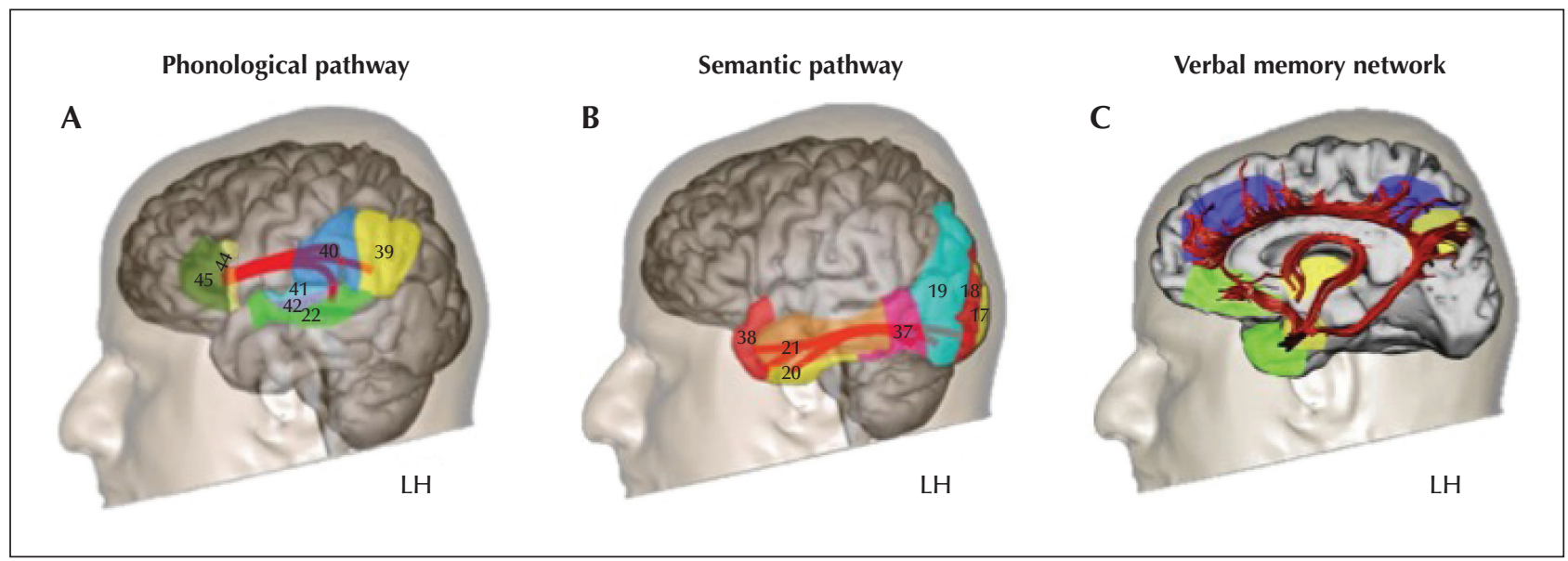

Figure 1. Illustration of the main association fascicles involved in language and verbal memory networks (adapted from Catani and Thiebaut de Schotten [2012] and Catani et al. [2013], with permission).

(A) Representation of the language network according to Mesulam (2000); the superior longitudinal fascicle (SLF) and the arcuate fascicle (Arc) are involved in the dorsal phonological pathway, described in the Duffau model (Duffau, 2008) and connect Broca's area to Wernicke's area, among others.

(B) The semantic ventral pathway for recognising or identifying objects and faces (Mesulam, 2000; Duffau et al., 2014); this concentrates inferior fascicles -the inferior longitudinal fascicle (ILF) and inferior fronto-occipital fascicle (IFOF)- that converge towards the temporal pole.

(C) Illustration of the memory-emotion network according to Mesulam (2000); this concerns a vast network involving the fornix (Fox), the cingulum (Cing), and the uncinate (Unc) fascicles. Usually, language-verbal memory networks are essentially lateralized in the left hemisphere $(\mathrm{LH})$.

considerable variability has been observed. Instead, according to Walhovd et al. (2014), the most plausible hypothesis is that myelin thickness variability is directly related to neuronal activity. A number of studies have shown that the myelin-producing precursors, the oligodendrocytes, have the capacity to detect and react to changes occurring in neuronal activity (Gibson et al., 2014).

Diffusion MRI enables in vivo non-invasive imaging of the microstructural characteristics of WM in the whole brain. If WM macrostructure appears to be relatively unchangeable, its microstructure, on the other hand, is plastic. Diffusion tensor imaging (DTI) carried out in young children shows that fibre bundle maturation:

- unfolds in tandem with child development;

- is associated with increased electrical transmission; - and appears to reflect the progress that a new-born child makes after birth (Dubois et al., 2012). Schlegel et al. (2012) observed microstructure plasticity in several WM bundles following intensive second-language learning. In elderly persons, the microstructural integrity of WM and executive capacity, such as speed information processing, are interrelated (for a review see Madden et al. [2012]).

These studies again suggest that myelination is directly related to neuronal activity (Walhovd et al., 2014). The myelin sheath-producing oligodendrocytes are the most metabolically active cells and thus the most vulnerable to metabolic lesions (Madden et al., 2012). Structural connectivity directly supports functional wiring (Hervé et al., 2013), but cognitive functioning also has a modulating effect on these connections. Microstructural changes can affect connection quality (strength or velocity) and at the same time, generation and elimination of synapses and connections (longterm potentiation or depression) are determined by the activity (synchronous/asynchronous discharges, or no discharges) that unfolds between various cells or on a broader scale, cortical regions.

\section{Structural connectivity and neurocognition of language and memory}

Recently developed neurocognitive models integrate structural connectivity in addition to functional data. These hodological models are based on the concept that cerebral processing results from the integration and potentiation of networks composed of parallel, albeit partially overlapping, sub-circuits (Duffau et al., 2013).

The well-described hodological model of language connectivity proposed by Duffau (2008) (revised by Duffau et al. [2014]) is developed from direct electrical subcortical stimulation and focuses on the role of various WM bundles in language operations. This model, which includes a dorsal and ventral pathway, is based on the concept that language function results from a coherent action of neuron populations which 
are largely distributed and inter-connected in parallel, constituting two main bidirectional pathways; a ventral semantic pathway and a dorsal phonological pathway. The dorsal phonological pathway directly connects the posterior temporal cortex to the posterior frontal cortex, via the arcuate fasciculus (Arc). When this WM bundle is stimulated (for language, in the dominant hemisphere) during a naming task, phonemic paraphasia is observed (Martino et al., 2013). The arcuate fasciculus thus appears to play a crucial role in phonological processing. The phonological pathway is likewise supported by the superior longitudinal fasciculus (SLF), which is lateral to the arcuate fasciculus and which underlies:

- the articulatory loop (inferior frontal cortex to supramarginal gyrus) involved in auditory and verbal working memory (SLF-a: anterior segment);

- and the discourse perception network, connecting the supramarginal gyrus and the posterior temporal regions (SLF-p: posterior segment). Stimulation of the SLF in the predominant hemisphere for language engenders articulatory disorders (Moritz-Gasser et al., 2013) induced by dysfunction of the executive language system that controls motor planning and flexibility (Kinoshita et al., 2015).

DTI tractography studies and anatomical dissections of the ventral semantic pathway reveal two partially overlapping branches (Sarubbo et al., 2016). A direct branch connects the occipital and temporal regions to prefrontal areas via the inferior fronto-occipital fasciculus (IFOF). This lexico-semantic pathway (in the left hemisphere for language) facilitates access to knowledge and concepts (Moritz-Gasser et al., 2013), as well as to semantic control (Whitney et al., 2012). An indirect branch supported by the inferior longitudinal fasciculus (ILF), of which the posterior segment connects the occipital to the occipito-temporal functional zones (visual form of objects), is involved in visual recognition and object identification (Mandonnet et al., 2009). Stimulation of the ventral semantic pathway triggers:

- semantic paraphasia in nearly $90 \%$ of cases during a visual object-recognition test;

- and verbal perseverations (Khan et al., 2014).

These cortico-cortical circuits appear to act mutually, in parallel and interactively, before joining up with the prefrontal cortex for integration of the final products of each of the subnetworks. It also appears that the functioning of this large distributed network is modulated by a striato-cortical loop that connects the frontal mesial structures to the head of the caudate nucleus. This loop plays a role in language control and particularly in selection, inhibition, and planning. In addition, a final common pathway leads to the last stage of speech production (Duffau, 2008; Duffau et al., 2014).
There is so far no clearly established hodological model for memory. However, a large fronto-parietotemporal network that involves the left hemisphere appears to be responsible for verbal memory (Alessio et al., 2013; Perrone-Bertolotti et al., 2015). In line with the aforementioned information concerning the ventral semantic pathway, the left ILF plays a major part in the memorization of verbal information (Shinoura et al., 2007). In addition, the uncinate fasciculus (Unc), a fronto-temporal connection, likewise plays a major role in the memory process (encoding and recognition, and autonoetic consciousness), mainly because resecting it severs the associative temporal cortex from the frontal cortex (Travers, 2008). Other WM fibres, namely the cingulate (Cing) and fornix (Fox) that are part of the Papez circuit, are involved in memory and learning (Metzler-Baddeley et al., 2017). In the medial temporal lobe, the connections between limbic structures, as well as between these structures and lateral temporal regions, allow for activity synergy between emotions, behavior, and memory, which are essential for the constitution of episodic memory traces (Catani et al., 2013).

These observations concerning the structural connectivity of language and memory processes confirm Mesulam's theories (figure 1). Based on "a lesion approach" and functional imaging, Mesulam (2000) described a number of large networks that are involved in cognition and behavior. One of these networks is the language network containing two epicentres: Broca's and Wernicke's areas (an object and face identification network that converges towards a crucial zone of the semantic system; the temporal pole) and an emotion and memory network (of which the epicentres are located in the internal temporal lobe; the amygdalohippocampic complex).

Language and verbal memory functions, which are inextricably bound up with intrinsic networks based on temporal hubs, are normally lateralized in the left hemisphere in healthy subjects (in terms of language for $95 \%$ and $80 \%$ of right and left-handed subjects, respectively) (Hervé et al., 2013). Likewise, whereas the right hemisphere plays an important role under certain conditions, such as in prosodic processing, when subjects are confronted with a complex task for which they lack the requisite expertise, or in the case of brain pathology (Baciu and Perrone-Bertolotti, 2015), the left hemisphere appears to be specialised for these functions. Beginning in early childhood, healthy subjects exhibit microstructural asymmetry of the left WM bundles, a phenomenon that appears to be the counterpart of the morphometric asymmetry found in cortical language areas (Dubois et al., 2009). 


\section{Structural connectivity and the specific role in focal epilepsy}

Focal epilepsy involves recurrent seizures induced by paroxystic and hyper-synchronous electrical discharges from groups of neurons involving a limited cortical region, the epileptogenic zone (EZ) (Fisher et al., 2017). However, the classic definition of the $E Z$ as "the necessary and sufficient cortical region which initiates seizures and the removal or disconnection of which stops seizures" seems to be insufficient. Indeed, focal-onset seizures are sometimes provoked by micro regions that are located at a distance from the zones where seizures normally originate (Stead et al., 2010). This also explains why a resection of the EZ, even complete, is not always sufficient to stop seizures. The concept of the EZ should be regarded more dynamically and from an integrative perspective, rather than a localized region responsible for seizures (de Curtis and Avoli, 2015). Indeed, focal epilepsy is increasingly being considered as a pathology of the networks of the brain (Engel, 2013) and the term "systemic epilepsy" is currently proposed (Avanzini et al., 2012).

Temporal lobe epilepsy (TLE) is the most common type of focal epilepsy, accounting for 70 to $80 \%$ of all adult cases (Jaimes-Bautista et al., 2015). TLE is often associated with unilateral mesial temporal sclerosis, which usually takes the form of hippocampal sclerosis (HS). More than $70 \%$ of TLE patients also present with hippocampal atrophy. One third of TLE patients have drug-resistant epilepsy and surgery is the only curative therapy which can stop the seizures (Bernasconi et al., 2011). Seizures and surgical resection may affect cognitive functioning and TLE patients often show cognitive deficits. Neuropsychological symptoms are usually mild to moderate given that significant anatomical and functional reorganization of cognitive networks may occur due to either chronic cerebral dysfunction or surgery.

With respect to the chronic pathological process, though transitory, epileptic seizures are excitotoxic (i.e. they are neurotoxic because of the high rate of excitatory neurotransmitter release; de Curtis and Avoli [2015]). In addition, repeated activation of synapses and interconnected networks during seizures induces various forms of neuronal plasticity (Bliss and Collingridge, 1993). Moreover, epileptic seizures may cause neuronal loss, followed by the development of new excitatory synapses and axonal sprouting. Molecular biological studies have shown that an episode of epileptic hyperactivity could activate as many as 1,000 genes (Pollard et al., 1996) that correspond to precursors of growth factors, leading to protein synthesis and dendritic development. Based on studies published over the past few decades and animal models, but also in humans with various forms of epilepsy, the constitution of neo-synapses has been described. In general, the majority of these newly constituted synapses are anatomically and functionally aberrant ("reactive plasticity" [Esclapez et al., 1999; Ben-Ari et al., 2008]), reflected by cognitive deficits.

The most frequent type of cognitive impairment in TLE concerns language and memory functions (Alessio et al., 2013). In a review, Hoppe et al. (2007) reported that $55 \%$ of all TLE patients suffered from episodic verbal or non-verbal memory impairments, and that $43 \%$ of them suffered from language impairments such as visual naming, verbal fluency, and comprehension deficits, suggesting here again that plasticity processes occur in epileptic patients, not without any consequences on cognitive functioning. The reorganization of cerebral networks has traditionally been studied using invasive methods, such as the Wada procedure (Wada, 1949) or stereo-electroencephalography (sEEG) (Bancaud and Talairach, 1973). However, recent developments of new neuroimaging technologies allow us to assess non-invasively the representation of cognitive networks. Brain networks and their plasticity can thus be considered using two main approaches: a functional approach (rest or task-related connectivity) and/or structural approach (anatomical connectivity). These two approaches are complementary and strongly related (Schmidt et al., 2014). Since resting networks of language and especially memory function are not yet clearly defined, this article will focus on the relationship between the reorganization patterns observed in task-related fMRI and structural connectivity. Moreover, under the neurocognitive perspective of focal and drug-resistant epilepsy, the links with cognition will also be addressed.

\section{Language and memory plasticity in focal epilepsy: a focus on TLE}

\section{Functional reorganization}

Functional imaging studies clearly show that atypical representations of language, which occur in a minority of the general population (Mazoyer et al., 2014), are far more prevalent in epileptic patients. The percentage of TLE subjects that exhibit atypical language representation is twice that of healthy individuals (Baciu and Perrone-Bertolotti, 2015). During seizurefree periods, three atypical patterns are observed: inter-hemispheric, crossed inter-hemispheric, and intra-hemispheric (figure 2).

Inter and intra-hemispheric functional differences for verbal memory are also more prevalent in TLE patients than in healthy individuals. The findings in this regard are analogous to those obtained in language studies. 

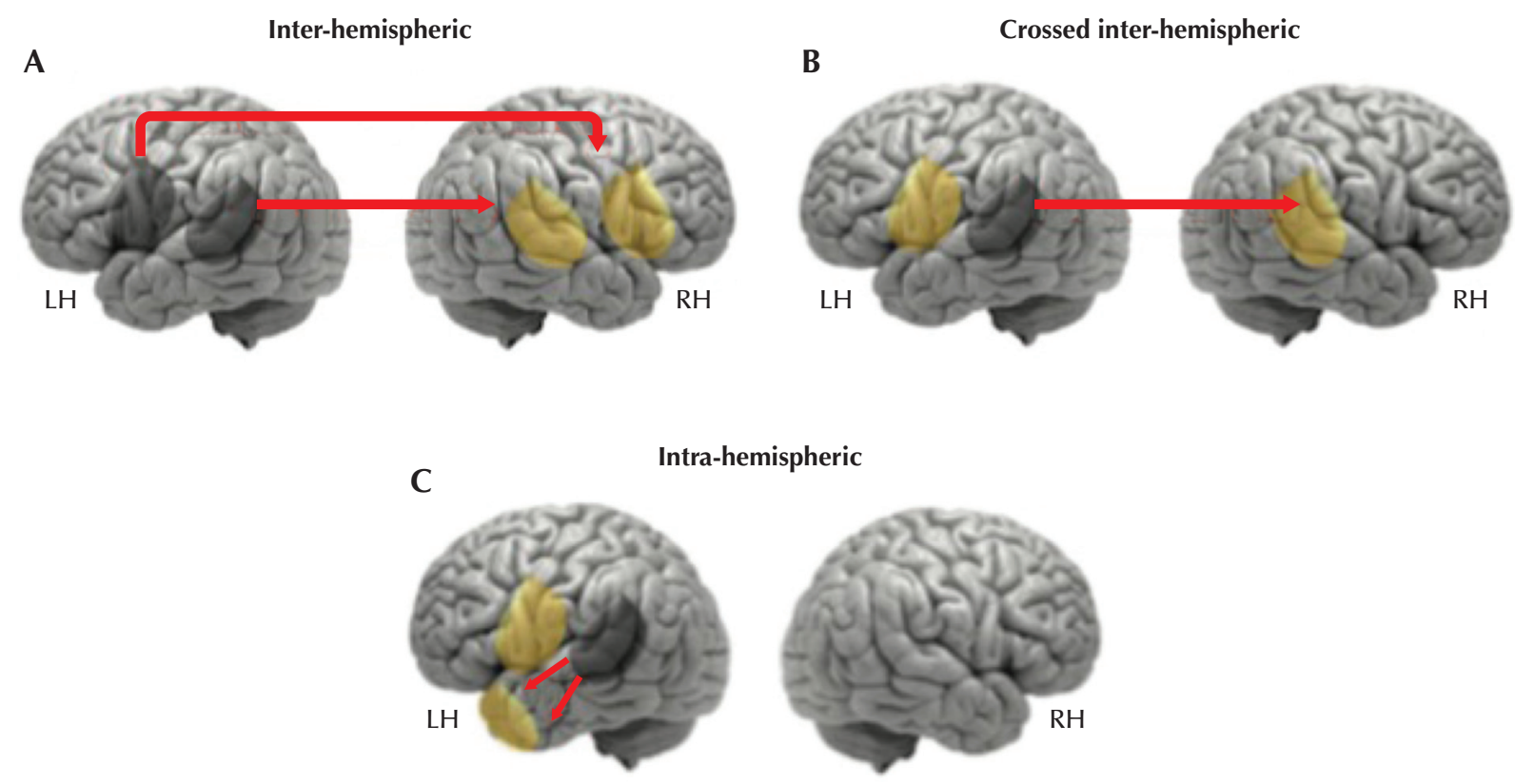

Figure 2. Illustration of the patterns of functional reorganization observed in epileptic patients (reproduced from Baciu and PerroneBertolotti [2015], with permission).

At the inter-hemispheric level, there is involvement or displacement of critical areas for language in the contralateral hemisphere (most often in homologous areas of the right hemisphere); this may be complete (A), or partial (B). At the intra-hemispheric level (C), there is additional recruitment or displacement of linguistic regions beyond the classic "eloquent areas" within the dominant hemisphere.

For example, Haag and Bonelli (2013) reported that in TLE patients:

- memory functions are transferred to homologous regions in the contralateral hemisphere (interhemispheric pattern);

- and/or at the intra-hemispheric level, with additional recruitment of ipsilateral frontal regions occurs;

- or such recruitment occurs at the extra-temporal level. Regardless of whether language or memory functions are studied, these atypical patterns appear to be related to poor performance in some TLE patients. Whereas in normal subjects, naming tasks triggers left hippocampal activation, in TLE patients, only the inferior frontal gyri and left-hemisphere resources are activated. This frontal "strategy" appears to be inefficient, given that these patients perform naming tasks sub-optimally (Bonelli et al., 2011). As with language, over-recruitment of frontal regions during memory tasks correlates with lower performance.

\section{Structural reorganization}

Foscolo et al. (2007) investigated the structure of the uncinate and inferior longitudinal fasciculi in TLE patients, based on their anatomical proximity to the epileptogenic zone. In line with the theory of the minimal common brain (lus et al., 2011), Foscolo and colleagues found no anatomical difference in fibre macrostructure (mean length and fibre bundle orientation) between TLE patients and a healthy control group. Thus, this again confirms that if there are any WM anomalies in patients with focal epilepsy, they are only detectable on a meso-microscale (microstructure). Indeed, in patients suffering from left TLE, DTI studies showed that the microscopic integrity of the uncinate and arcuate fascicules is impaired, along with the fornix and cingulum (Concha et al., 2005). Hence the bundles involved in language and memory are typically impaired in these patients; a fact attested to by their cognitive deficits.

Language and/or memory impairments in TLE patients appear to be directly related to the integrity of the WM bundles that traverse the temporal lobe. McDonald et al. (2008) found a strong association between left arcuate fasciculus integrity and patients' naming abilities. In regard to memory in TLE patients, Riley et al. (2010) observed that the integrity of the uncinate fasciculus and the left inferior longitudinal fasciculus is correlated with delayed verbal memory performance, whereas fornix integrity is related to short-term memory scores. Even more interestingly, Kucukboyaci et al. (2014) compared volumetric DTI and MRI in terms 
of naming deficits observed in TLE patients. Neither cortical thickness nor the differences between grey matter and WM in fronto-temporal regions provide a satisfactory explanation for these patients' performance. A contrario, the diffusion parameters correlated strongly with performance, even after controlling for hippocampus size. Hence, the correlation between cerebral impairments and cognitive deficits in TLE patients appears to be closely linked to WM.

In accordance with the concept of plastic and activitydependent microstructural connectivity, Ellmore et al. (2010) showed that the structural asymmetry index based on DTI data for language-related tracts can be used to estimate, with a significant predictive power, the functional lateralization of linguistic capacities in TLE patients. Furthermore, Dinkelacker et al. (2016) evoked in their review a hyper-connectivity phenomenon (increase in structural connectivity for certain subcortical zones in patients with epilepsy compared to healthy subjects) that could reflect functional compensation.

Although the foregoing data already suggest this, further studies focusing specifically on the links between structural connectivity and reorganization of language and memory (e.g. atypical right fMRI lateralization/inter-hemispherical shift) are needed. These studies will thus confirm the postulate of a close interrelation between functional reorganizations and changes in terms of structural connections in epileptic patients, and will further verify or invalidate certain assumptions, such as commissural fibre impairment as a cause or a main contributing factor of the interhemispherical shifting observed with high prevalence in these patients (the transcallosal inhibition reduction hypothesis; e.g. Baciu and Perrone-Bertolotti [2015]). Basically, DTI studies performed on patients with focal and drug-resistant epilepsy clearly reveal a global change in WM microstructure. Although epilepsy is traditionally regarded as a cortex disorder, WM structures (including those at a distance from the primary epileptogenic zone) are impacted in focal epilepsy patients. WM regions that are contralateral to the presumed EZ are affected by these events (Concha et al., 2005; van Eijsden et al., 2011).

To take again the case of TLE patients, diffusion abnormalities are not confined to the epileptogenic temporal lobe, but also involve a vast network comprising various associations, projections, and commissural bundles. Diffusion parameter modification is observed during seizure-free periods and is not limited to lesional epilepsies (in the presence of HS, for example), but also occurs in non-lesional epilepsy (cryptogenic epilepsy in which no lesion is detected by conventional MRI [Rodrigo et al., 2008]). Another study (Keller et al., 2012) found that diffusion anomalies correlate with the duration of epilepsy; a phenomenon the authors viewed as a gradual degenerative process resulting from chronic seizures. Thus, here too, DTI studies showed that epilepsy cannot be regarded solely as a focal cortical pathology with a clearly unilateral lesion.

The existence of diffuse and bilateral changes in the WM of TLE patients with drug resistance is thus well documented. DTI and histological studies of these patients reveal axonal loss (Dingledine et al., 2014), reduced axonal diameters, and myelin sheath defects in the surviving axons of the presumed epileptogenic zone (Rodríguez-Cruces and Concha, 2015), however, an important debate concerns the spatial dynamics of impairments. Indeed, the intensity and nature of damage between WM bundles directly connected to the epileptogenic zone and those at a greater distance from it are not yet clearly defined.

This debate and controversy globally derive from two theories, one of "propagation" and another one of "initiation". Regarding the propagation theory, the generalized diffusion anomalies are symmetrical and of equal scope inter-hemispherically (e.g. Yu et al. [2008]), whereby this degeneration appears to be related to co-occurring commissural fibre impairments (Foscolo et al., 2007). The corpus callosum is particularly vulnerable to epilepsy (Caligiuri et al., 2016) and is often involved in seizure transmission through the hemispheres (and in relation to generalization). In TLE patients, for example, seizures propagate to the frontal lobe almost every time (mainly via the uncinate fasciculus) and are very often propagated to the other hemisphere (Mayanagi et al., 1996). Thus, the frontal lobe appears to serve as the access point for seizure propagation by the corpus callosum. In line with the symmetry theory, the nature of the changes in the ipsilateral epileptogenic hemisphere should be identical, as these changes are related to seizure propagation (Imamura et al., 2016). These observations suggest that contralateral epileptogenic hemisphere changes are a direct and primary effect of epileptic activity.

Regarding the initiation theory (Ahmadi et al., 2009; Concha et al., 2010), such changes are far more pronounced in the ipsilateral hemisphere in local epilepsy networks (epileptigenic zone). Based on a meta-analysis of 13 studies, Otte et al. (2012) compared the integrity of various WM tissues in TLE patients, according to their degree of connection with the epileptogenic temporal lobe; in line with the theory of initiation, the deterioration of directly connected structures was greater than that of the most distant structures, such as contralateral fibres. Riley et al. (2010) found a "centrifugal decrease" of deficits with regards to the epileptogenic zone, despite diffuse 
WM changes. According to these authors, the nature of impairments also varies. The variance observed in local networks (around the EZ) is attributable to primary chronic degeneration, whereas secondary damage (minor fibre loss or myelin degradation) is observed in other affected bundles; the authors associate the latter with phenomena of disconnections or diaschisis. In this regard, changes in the contralateral epileptogenic hemisphere are regarded as an indirect and secondary effect of seizures.

\section{Conclusions}

Apart from the theoretical benefits of addressing the issue of the spatial dynamics of impairments, the structural connectivity approach will also have major benefits for clinical practice. Further structural connectivity studies in patients with focal and drug-resistant epilepsy -in particular via non-invasive diffusion imaging- will provide more information on what is happening at a distance from the presumed epileptogenic zone. Given that a certain amount of subcortical plasticity exists, this approach may well allow biomarkers to be identified that can be used, for example, to localize the region that needs to be removed (e.g. epileptogenic networks), as well as functional regions that must not be impacted. Thus, it may well be possible for this technique to be integrated into clinical practice for preoperative evaluations of epileptic patients with drug-resistance, as a guide to surgical decision-making in order to improve the resection versus function balance.

In order to fully take advantage of the focal nature of an epileptic condition, and in particular the TLE model for the study of language-memory brain reorganization (the temporal lobe being deeply involved in language and memory functions), a multimodal approach should always be used. A major work now concerns the identification of the potential relationships between:

- impaired WM belonging to language and memory structural networks based on DTI analyses;

- and functional reorganization patterns that come to light via task and/or rest-related fMRI for language memory functional networks.

The results of these investigations should be interpreted in light of the scores obtained from the different sub-processes of these cognitive functions and of the clinical modulating factors (lateralization/location of EZ, epilepsy duration, antiepileptic drugs, etc.).

This multimodal approach will help to develop new integrated neurocognitive models for use in clinical settings. A multimodal study of brain plasticity in these patients will allow biomarkers to be identified that are specific to the chronic impairments associated with epilepsy.

When related to the cognitive scores, these biomarkers will enable us to define the various reorganization profiles, e.g. efficient reorganization, as opposed to less efficient reorganization. Some profiles could be determined in both pre- and post-surgical phases (follow-up) in order to detect longitudinal modifications of features of structural connectivity linked to acute or subacute plasticity in the early stages, followed by those corresponding to long-term recovery. In addition, comparisons between networks observed after successful (cessation or significant reduction of seizures) and unsuccessful (no effect on seizures) surgery can potentially be used to identify regular patterns of persistent and problematic epileptic networks. This would be useful to improve guidance towards supplementary therapeutic interventions.

Unfortunately, the study of structural connectivity in the post-surgical phase is still lacking and must be developed. The ultimate goal of this type of approach will be to acquire the capacity to predict postoperative outcomes (clinical, cognitive, and cerebral prognoses) solely based on preoperative characteristics, by using the above-mentioned biomarkers as a basis. Moreover, inasmuch as structural connectivity appears to be strongly correlated with cognitive abilities, it will be a key factor that should be taken into account in order to describe and understand the mechanisms related to the efficiency of brain reorganization; it will also help us to develop fully integrated and dynamic neurocognitive models (including hodological models).

Indeed, one of the greatest challenges facing neuroscience today is the integration of multimodal data, with the goal of gaining an understanding of the neuronal processes and networks that support cognition and behavior. Structural connectivity engenders new insights concerning brain networks. If the relationship between these networks and brain cognition and function is elucidated, these insights could potentially set the stage for the development of a complete and integrated connectome for epileptic pathology. $\square$

\section{Supplementary data.}

Summary didactic slides are available on the www.epilepticdisorders.com website.

\section{Disclosures.}

None of the authors have any conflict of interest to declare.

\section{References}

Ahmadi ME, Hagler D, McDonald CR, et al. Side matters: diffusion tensor imaging tractography in left and right temporal lobe epilepsy. Am J Neuroradiol 2009; 30:1740-7. 
Alessio A, Pereira FR, Sercheli MS, et al. Brain plasticity for verbal and visual memories in patients with mesial temporal lobe epilepsy and hippocampal sclerosis: an fMRI study. Hum Brain Mapp 2013; 34: 186-99.

Avanzini G, Manganotti P, Meletti S, et al. The system epilepsies: a pathophysiological hypothesis. Epilepsia 2012; 53: 771-8.

Baciu M, Perrone-Bertolotti M. What do patients with epilepsy tell us about language dynamics? A review of $\mathrm{fMRI}$ studies. Rev Neurosci 2015; 26:323-41.

Bancaud J, Talairach J. Methodology of stereo EEG exploration and surgical intervention in epilepsy. Rev Otoneuroophtalmol 1973; 45: 315.

Ben-Ari Y, Crepel V, Represa A. Seizures beget seizures in temporal lobe epilepsies: the boomerang effects of newly formed aberrant kainatergic synapses. Epilepsy Curr 2008; 8: 68-72.

Bernasconi A, Bernasconi N, Bernhardt BC, et al. Advances in MRI for 'cryptogenic' epilepsies. Nat Rev Neurol 2011; 7: 99-108.

Bliss TV, Collingridge GL. A synaptic model of memory: longterm potentiation in the hippocampus. Nature 1993;361: $31-9$.

Bonelli SB, Powell R, Thompson PJ, et al. Hippocampal activation correlates with visual confrontation naming: $f M R I$ findings in controls and patients with temporal lobe epilepsy. Epilepsy Res 2011; 95: 246-54.

Caligiuri ME, Labate A, Cherubini A, et al. Integrity of the corpus callosum in patients with benign temporal lobe epilepsy. Epilepsia 2016; 57: 590-6.

Catani M, Thiebaut de Schotten M. Atlas of Human Brain Connections. Oxford University Press, 2012.

Catani M, Dell'Acqua F, De Schotten MT. A revised limbic system model for memory, emotion and behaviour. Neurosci Biobehav Rev 2013; 37: 1724-37.

Concha L, Beaulieu C, Gross DW. Bilateral limbic diffusion abnormalities in unilateral temporal lobe epilepsy. Ann Neurol 2005; 57: 188-96.

Concha L, Livy DJ, Beaulieu C, et al. In vivo diffusion tensor imaging and histopathology of the fimbria-fornix in temporal lobe epilepsy. J Neurosci 2010; 30: 996-1002.

de Curtis M, Avoli M. Initiation, propagation, and termination of partial (focal) seizures. Cold Spring Harb Perspect Med 2015; 5: a022368.

Dingledine $\mathrm{R}$, Varvel NH, Dudek FE. When and how do seizures kill neurons, and is cell death relevant to epileptogenesis? Adv Exp Med Biol 2014; 813: 109-22.

Dinkelacker V, Dupont S, Samson S. The new approach to classification of focal epilepsies: epileptic discharge and disconnectivity in relation to cognition. Epilepsy Behav 2016; 64: 322-8.

Dubois J, Hertz-Pannier L, Cachia A, et al. Structural asymmetries in the infant language 20 and sensori-motor networks. Cereb Cortex 2009; 19: 414-23.
Dubois J, Dehaene-Lambertz G, Mangin J-F, et al. Neurophysiologie clinique: développement cérébral du nourrisson et imagerie par résonance magnétique. Neurophysiol Clin Neurophysiol 2012; 42: 1-9.

Duffau $\mathrm{H}$. The anatomo-functional connectivity of language revisited. Neuropsychologia 2008; 46: 927-34.

Duffau H. A two-level model of interindividual anatomofunctional variability of the brain and its implications for neurosurgery. Cortex 2017; 86: 303-13.

Duffau H, Herbet G, Moritz-Gasser S. Toward a pluricomponent, multimodal, and dynamic organization of the ventral semantic stream in humans: lessons from stimulation mapping in awake patients. Front Syst Neurosci 2013; 7: 44.

Duffau H, Moritz-Gasser S, Mandonnet E. A re-examination of neural basis of language processing: proposal of a dynamic hodotopical model from data provided by brain stimulation mapping during picture naming. Brain Lang 2014;131: 1-10.

Ellmore TM, Beauchamp MS, Breier JI, et al. Temporal lobe white matter asymmetry and language laterality in epilepsy patients. Neuroimage 2010; 49: 2033-44.

Engel J. Seizures and Epilepsy. Oxford University Press, 2013. Esclapez M, Hirsch JC, Ben-Ari Y, et al. Newly formed excitatory pathways provide a substrate for hyperexcitability in experimental temporal lobe epilepsy. J Comp Neurol 1999; 408: 449-60.

Fisher RS, Cross JH, French JA, et al. Operational classification of seizure types by the International League Against Epilepsy: Position Paper of the ILAE Commission for Classification and Terminology. Epilepsia 2017; 58:522-30.

Foscolo S, Periot O, Schmitt E, et al. Les faisceaux uncine et longitudinal inférieur dans l'épilepsie temporale: étude préliminaire en tractographie. J Neuroradiol 2007; 34: 24 .

Gibson EM, Purger D, Mount CW, et al. Neuronal activity promotes oligodendrogenesis and adaptive myelination in the mammalian brain. Science 2014; 344: 1252304.

Glickstein M, Berlucchi G. Classical disconnection studies of the corpus callosum. Cortex 2008; 44: 914-27.

Haag A, Bonelli S. Clinical application of language and memory fMRI in epilepsy. Epileptologie 2013;30: 101-8.

Hau J, Sarubbo S, Perchey G, et al. Cortical terminations of the inferior fronto-occipital and uncinate fasciculi: anatomical stem-based virtual dissection. Front Neuroanat 2016; 10: 58.

Hervé PY, Zago L, Petit L, et al. Revisiting human hemispheric specialization with neuroimaging. Trends Cogn Sci 2013; 17:69-80.

Hoppe C, Elger CE, Helmstaedter C. Long-term memory impairment in patients with focal epilepsy. Epilepsia 2007; 48: 26-9.

Imamura H, Matsumoto R, Takaya S, et al. Network specific change in white matter integrity in mesial temporal lobe epilepsy. Epilepsy Res 2016; 120:65-72. 
Ius T, Angelini E, Thiebaut de Schotten M, et al. Evidence for potentials and limitations of brain plasticity using an atlas of functional resectability of WHO grade II gliomas: towards a "minimal common brain". Neuroimage 2011; 56: 992-1000.

Jaimes-Bautista AG, Rodríguez-Camacho M, Martínez-Juárez $\mathrm{IE}$, et al. Semantic processing impairment in patients with temporal lobe epilepsy. Epilepsy Res Treat 2015;2015: 1-8.

Keller SS, Schoene-Bake JC, Gerdes JS, et al. Concomitant fractional anisotropy and volumetric abnormalities in temporal lobe epilepsy: cross-sectional evidence for progressive neurologic injury. PloS One 2012; 7: e46791.

Khan $\mathrm{OH}$, Herbet G, Moritz-Gasser S, Duffau H. The role of left inferior fronto-occipital fascicle in verbal perseveration: a brain electrostimulation mapping study. Brain Topogr 2014; 27: 403-11.

Kinoshita M, de Champfleur NM, Deverdun J, et al. Role of fronto-striatal tract and frontal aslant tract in movement and speech: an axonal mapping study. Brain Struct Funct 2015; 220: 3399-412.

Kiran S. What is the nature of poststroke language recovery and reorganization? ISRN Neurol 2012; 2012: 1-13.

Kucukboyaci N, Kemmotsu N, Leyden K, et al. Integration of multimodal MRI data via PCA to explain language performance. Neurolmage Clin 2014; 5: 197-207.

Lomber SG, Meredith MA, Kral A. Cross-modal plasticity in specific auditory cortices underlies visual compensations in the deaf. Nat Neurosci 2010;13:1421-7.

Lunven M, Thiebaut de Schotten M, Bourlon C, et al. White matter lesional predictors of chronic visual neglect: a longitudinal study. Brain 2015; 138: 746-60.

Madden DJ, Bennett IJ, Burzynska A, et al. Diffusion tensor imaging of cerebral white matter integrity in cognitive aging. Biochim Biophys Acta 2012; 1822: 386-400.

Mandonnet E, Gatignol P, Duffau H. Evidence for an occipitotemporal tract underlying visual recognition in picture naming. Clin Neurol Neurosurg 2009; 111:601-5.

Martino J, Hamer PC, Berger MS, et al. Analysis of the subcomponents and cortical terminations of the perisylvian superior longitudinal fasciculus: a fiber dissection and DTI tractography study. Brain Struct Funct 2013;218: 105-21.

Mayanagi Y, Watanabe E, Kaneko Y. Mesial temporal lobe epilepsy: clinical features and seizure mechanism. Epilepsia 1996; 37:57-60.

Mazoyer B, Zago L, Jobard G, et al. Gaussian mixture modeling of hemispheric lateralization for language in a large sample of healthy individuals balanced for handedness. PLOS One 2014; 9: e101165.

McDonald CR, Ahmadi ME, Hagler DJ, et al. Diffusion tensor imaging correlates of memory and language impairments in temporal lobe epilepsy. Neurology 2008; 71: 1869-76.

Mesulam MM. Principles of Behavioral and Cognitive Neurology. Oxford University Press, 2000.
Metzler-Baddeley C, Foley S, De Santis S, et al. Dynamics of white matter plasticity underlying working memory training: multimodal evidence from diffusion MRI and $\mathrm{T} 2$ relaxometry. J Cogn Neurosci 2017; 29: 1509-20.

Moritz-Gasser S, Herbet G, Duffau H. Mapping the connectivity underlying multimodal (verbal and non-verbal) semantic processing: a brain electrostimulation study. Neuropsychologia 2013; 51: 1814-22.

Otte WM, van Eijsden P, Sander JW, et al. A meta-analysis of white matter changes in 22 temporal lobe epilepsy as studied with diffusion tensor imaging. Epilepsia 2012; 53:659-67.

Perrone-Bertolotti M, Girard C, Cousin E, et al. NEREC, an effective brain mapping protocol for combined language and long-term memory functions. Epilepsy Behav 2015; 53:140-8.

Pollard H, Bugra K, Khrestchatisky M, et al. Seizure-induced molecular changes, sprouting and synaptogenesis of hippocampal mossy fibers. Epilepsy Res Supp/ 1996; 12: 355.

Riley JD, Franklin DL, Choi V, et al. Altered white matter integrity in temporal lobe epilepsy: association with cognitive and clinical profiles. Epilepsia 2010; 51:536-45.

Rodrigo S, Oppenheim C, Chassoux F, et al. Language lateralization in temporal lobe epilepsy using functional MRI and probabilistic tractography. Epilepsia 2008; 49:1367-76.

Rodríguez-Cruces R, Concha L. White matter in temporal lobe epilepsy: clinico-pathological correlates of water diffusion abnormalities. Quant Imaging Med Surg 2015; 5: 264.

Sarubbo S, De Benedictis A, Merler S, et al. Structural and functional integration between dorsal and ventral language streams as revealed by blunt dissection and direct electrical stimulation. Hum Brain Mapp 2016; 37:3858-72.

Schlegel AA, Rudelson JJ, Peter UT. White matter structure changes as adults learn a second language. J Cogn Neurosci 2012; 24: 1664-70.

Schmidt R, Verstraete E, Reus MA, et al. Correlation between structural and functional connectivity impairment in amyotrophic lateral sclerosis. Hum Brain Mapp 2014; 35: 4386-95.

Shinoura N, Suzuki Y, Tsukada M, et al. Impairment of inferior longitudinal fasciculus plays a role in visual memory disturbance. Neurocase 2007; 13:127-30.

Sporns O. Structure and function of complex brain networks. Dialogues Clin Neurosci 2013;15: 247.

Stead M, Bower M, Brinkmann BH, et al. Microseizures and the spatiotemporal scales of human partial epilepsy. Brain 2010; 133: 2789-97.

Tomassy GS, Berger DR, Chen HH, et al. Distinct profiles of myelin distribution along single axons of pyramidal neurons in the neocortex. Science 2014;344:319-24.

Travers N. L'étude micro-anatomique des fibers du faisceau unciné et ses implications dans la chirurgie fronto-temporoinsulaire. Académie Natl Chir 2008; 7:31-41.

Van Eijsden P, Otte WM, Saskia van der Hel W, et al. In vivo diffusion tensor imaging and ex vivo histologic characterization of white matter pathology in a post-status epilepticus model of temporal lobe epilepsy. Epilepsia 2011; 52: 841-5. 
Wada J. A new method of determining the side of cerebral speech dominance: a preliminary report on the intracarotid injection of sodium amytal in Man. Igaku Seibutsugaki $1949 ; 14: 221-2$.

Walhovd KB, Johansen-Berg $\mathrm{H}$, Karadottir RT. Unraveling the secrets of white matter- bridging the gap between cellular, animal and human imaging studies. Neuroscience 2014; 276:2-13.
Whitney C, Kirk M, O'Sullivan J, et al. Executive semantic processing is underpinned by a large-scale neural network: revealing the contribution of left prefrontal, posterior temporal, and parietal cortex to controlled retrieval and selection using TMS. J Cogn Neurosci 2012; 24: 133-47.

Yu A, Li K, Li L, et al. Whole-brain voxel-based morphometry of white matter in medial temporal lobe epilepsy. Eur J Radiol 2008; 65: 86-90.

\section{TEST YOURSELF}

(1) What is an "hodological model"?

(2) What does the term "minimal subcortical plasticity" mean?

(3) With respect to the nature and degree of white matter alteration as a result of epileptic activity, explain the theory of initiation.

Note: Reading the manuscript provides an answer to all questions. Correct answers may be accessed on the website, www.epilepticdisorders.com, under the section "The EpiCentre". 